\title{
STELLAR ACTIVITY CYCLES: WHY DO WE NEED IN THE LONG-TERM MEASUREMENTS OF THE MAGNETIC FIELD?
}

\author{
S.I. Plachinda ${ }^{2}$, V.V. Butkovskaya ${ }^{1}$ \\ ${ }^{1}$ Main Astronomical Observatory of National Academy of Sciences, \\ Kyiv, Ukraine,psi1951@yahoo.com \\ ${ }^{2}$ Crimean Astrophysical Observatory, Nauchny, Crimea
}

\begin{abstract}
Solution of the two important problems requires a study of long-term behavior of the magnetic field on stars with nondegenerate matter: firstly, to define the role of the magnetic field on different evolutionary stages of stars; secondly, to know the influence of the star's activity on space weather in the habitable zone. This brief review summarizes the first results of the long-term magnetic field measurements on selected stars.
\end{abstract}

Key words: stars: activity - stars: late-type - stars: magnetic fields.

\section{Introduction}

Today we have a wide range of outstanding issues associated with the magnetization of the stellar matter: the origin of the stellar magnetism, the role of the magnetic field in the star formation and evolution, their rotational structure, mass loss, coronal activity and others activity phenomena.

Stellar activity cycles like the solar cycles have been found almost half a century ago. During this period, researchers have crystallized a number of important problems: measuring the duration of the activity cycles, the interplay between activity cycles, evolutionary status and stellar rotation, the variability of activity cycle duration and multicycles, and so on. Despite the facts that the regular, generally photometric, monitoring of some objects is carried out for decades, and the long-time datasets obtained by the space telescopes allow to discover short (0.5-6 years) cycles of activity for thousands of stars, the most of the task remain unsolved.

We present here the brief review of the first results of long-term measurements of the magnetic field on the selected stars.

\section{Long-term variability cycles of selected stars}

Radic et al. (1998) have found that the younger, more active stars "tend to become fainter as their HK emission increases, whereas the older, less active stars tend to become brighter as their HK emission increases, as the Sun does during its activity cycle."

The Sun. The absolute values of the General Magnetic Field (GMF) of the Sun as a star varies with the phase of the 11-year sunspot cycle. Today it is well known that in an epoch of the maximum of the solar activity (a maximal number of sunspots, a maximal flux in the chromospheric Ca II HK emission, a maximal visual photometric brightness, and so on) the GMF reaches its maximum $(\sim \pm 2 \mathrm{G})$ and in an epoch of the minimum solar activity GMF reaches its minimum $(\sim \pm 0.2 \mathrm{G})$. In other words, the variability of the GMF of the Sun as a star correlates with the phase of the sunspot cycle.

61 Cyg A. First results of the investigation of longterm magnetic field behavior on the old solar-like Kdwarf star 61 Cyg A was published by Saikia et al. (2016). 61 Cyg A shows Ca II emission periodicity in the time interval of about 7.3 years (Baliunas et al. 1995). But in spite of the above mentioned conclusion by Radic et al. (1998) in the case of 61 Cyg A Saikia et al. (2016) supposed a weak anti-correlation between the absolute value of the longitudinal magnetic field $\left|B_{l}\right|$ and the $S$-index of the calcium chromospheric emission.

$\beta$ Aql. For the next stage in the evolution of solarlike stars, convective subgiants, first long-term measurements of the longitudinal magnetic field have been performed by Butkovskaya et al. (2017). The authors have carried out the spectropolarimetric observations of $\beta \mathrm{Aql}\left(V_{\mathrm{mag}}=3.7, \mathrm{G} 8 \mathrm{IV}-\mathrm{V}\right)$ over 51 nights from 1997 to 2015 . The results of this investigation is shown in Figure 1.

The upper panel of Figure 1 shows averaged in 10 bins $V$-brightness, which are phased with suspected activity period of about $969 \mathrm{~d}$. The photometric data was taken from the All Sky Automated Survey (ASAS) and includes 344 measurements.

In the bottom panel of Figure 1 the total range of magnetic field variation, $\Delta B=B_{\max }-B_{\min }$, folded in phase with the $969 \mathrm{~d}$ period are presented. One can 


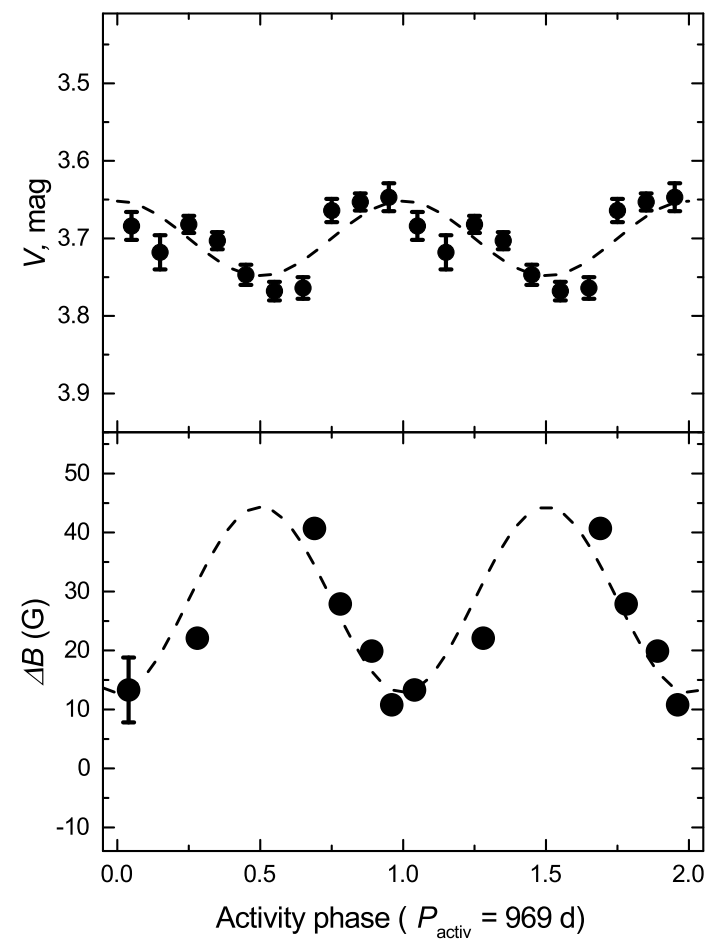

Figure 1: Upper panel: The $V$-brightness averaged in 10 bins phased with the 969 d activity period. The least-square sinusoidal fit is shown by the dashed line. Bottom panel: The range of magnetic field $\Delta B$ calculated using the magnetic field values falling in the same phases of the activity period. The $\mathrm{SD}=5.5 \mathrm{G}$ of these $\Delta B$ values from the fitting curve is shown as an error bar around the first value. The best least-square sinusoidal fit to $\Delta B$ is shown by the dashed line.

see that the maximum of $\Delta B$ fitting curve coincides in phase with the brightness minimum.

Vega. For more than six decades Vega has been accepted as a standard star in the near infrared, optical, and ultraviolet spectrum bands. Vasil'ev et al. (1989a) shows that the flux density E5556 produced by the emission of Vega at the wavelength $\lambda=5556 \AA$, varies with a period of $23-25$ years. This variability has been investigated in detail by Merezhin (2001), but the results of his study remained unrecognized. Butkovskaya et al. (2011) have analyzed their long-term spectral data and have verified the long-term variability cycle of Vega. They have found the cycle duration of $7697 \pm$ $157 \mathrm{~d}$ (21.28 year) and have concluded that the spectral and photometric standard Vega is a variable star. However, the physical mechanism of the long-term periodicity should be investigated. Also, the question "Does the magnetic field of Vega vary during the 21year period?" should be answered.

\section{Discussion and Conclusions}

In contradiction with the solar correlations of the brightness, Ca II HK chromospheric emission and the range of the magnetic field variation, $61 \mathrm{Cyg} \mathrm{A}$ shows a weak anticorrelation between the cyclic magnetic field variation and $\mathrm{Ca}$ II $\mathrm{HK}$ chromospheric emission and $\beta$ Aql shows the anticorrelation between the cyclic magnetic field variation and $V$-brightness.

On the other hand, the rapid increase in the number of discovered planetary systems strengthens the importance of the question of space weather on planets in the Habitable Zone (HZ). The possibility of origin of life on a planet in the HZ depends strongly on the magnetic activity of the hosting star whose activity level depends on the phase of the Hale cycle.

"The HZ is the circumstellar region inside which a terrestrial planet can hold permanent liquid water on its surface" (Selsis et al., 2007). NASA: "Conditions on the Sun and in the solar wind, magnetosphere, ionosphere and thermosphere that can influence the performance and reliability of space-borne and ground-based technological systems and can endanger human life or health."

Planets in the HZ of active stars can be exposed to high levels of X-ray and UV radiation and high-energy particle fluxes from the stellar wind or coronal mass ejections. Such high emissions are the result of the stellar magnetic activity and they can induce important thermal and non-thermal atmospheric losses to space, potentially able to strip the whole atmosphere. Therefore, to study the impact of the hosting star activity on the magnetosphere of planets the knowledge of the range of variability of the magnetic field of the hosting star is required.

As the duration of the activity cycles in stars of different spectral classes can reach several decades, long-term spectropolarimetric observations are required to detect these cycles and to investigate cyclical changes of the magnetic field.

\section{References}

Baliunas S.L., Donahue R.A., Soon W.H. et al.: 1995, ApJ, 438, 269.

Butkovskaya V., Plachinda S., Valyavin G. et al.: 2011, Astron. Nachr., 332, 956.

Butkovskaya V.V., Plachinda S.I., Bondar' N.I., Baklanova D.N.: 2017, Astron. Nachr., in press.

Merezhin V.P.: Vega. Analiz i interpretatsiya dannykh nablyuenii (Vega. Analysis and Interpretation for Observations), Kazan: Fen, 2001.

Radick R.R., Lockwood G.W., Skiff B.A. et al.: 1998, ApJSS, 118, 239.

Saikia S.B., Jeffers S.V., Morin J. et al.: 2016, $A \& A$, 594, A29.

Selsis F., Kasting J.F., Levrard B. et al.: 2007, $A \mathscr{E} A$, 476, 1373.

Vasil'ev, I.A. et al.: 1989, IBVS, 3308. 